\title{
Intravitreal injection of tissue plasminogen activator as treatment for an occluded pars plana glaucoma tube
}

\author{
Irena Tsui \\ Suzanna Airiani \\ Angie Wen \\ Tarek El-Sawy \\ Howard F Fine \\ Peter JG Maris Jr \\ Department of Ophthalmology, \\ Columbia University, New York, \\ NY, USA
}

Correspondence: Peter JG Maris Jr Edward S Harkness Eye Institute, 635 West I65th St, Box 34, New York, NY 10032, USA

$\mathrm{Tel}+|2| 23421190$

Fax + I 2123054863

Email pmaris@msn.com

\begin{abstract}
Implanting glaucoma tubes through the pars plana in the setting of a corneal transplant is becoming more common, and there are unique problems associated with such a procedure. A 42-year-old man with multiple previous eye surgeries presented with a nonfunctioning pars plana glaucoma tube. There was no view to the tube tip, but it was presumed to be clogged with fibrin. Intravitreal tissue plasminogen activator (tPA) was injected through the pars plana which resulted in intraocular pressure control without further surgery. This new application of intravitreal tPA has not been reported previously. Future research should investigate the optimal effective and safe dose of intravitreal tPA injection to relieve such occlusions.
\end{abstract}

Keywords: tissue plasminogen activator (tPA), Ahmed Glaucoma Valve

\section{Introduction}

Penetrating keratoplasty (PKP) with concurrent anterior chamber placement of a glaucoma tube shunt is associated with a high rate of corneal decompensation. ${ }^{1}$ Recently, the Cornea Glaucoma Implant Study Group demonstrated that in patients requiring a corneal transplant and a glaucoma tube shunt, the incidence of graft failure and immunologic rejection was decreased with pars plana insertion of the tube. ${ }^{2}$ We describe a novel application of tissue plasminogen activator (tPA) in the management of an occluded pars plana glaucoma drainage device.

\section{Case report}

A 59-year-old African American male had a history of keratoconus with two prior PKPs, cataract extraction with lens implantation, and a failed trabeculectomy in his left eye. He presented with an opaque, vascularized cornea and secondary angle-closure glaucoma. His vision was hand motions and intraocular pressure (IOP) was $40 \mathrm{mmHg}$ by Tonopen (Reichert, Depew, NY). Under general anesthesia, he underwent elective combined Eckardt temporary keratoprosthesis, pars plana vitrectomy, placement of an Ahmed Glaucoma Valve (New World Medical, Rancho Cucamonga, CA) through the pars plana and PKP.

On the first postoperative day, the IOP was $32 \mathrm{mmHg}$, the area over the Ahmed plate was flat, and the anterior chamber had diffuse fibrin formation. The following day, the IOP climbed to $48 \mathrm{mmHg}$ despite oral acetazolamide as well as dorzolamide hydrochloride $2 \%$, timolol maleate $0.5 \%$, and brimonidine tartrate $0.15 \%$ eyedrops. Suspecting a kink in the tube or vitreous incarceration, the patient was returned to the operating room where the tube was flushed easily and was repositioned through a new sclerostomy along the pars plana.

The IOP improved initially, but by one week rose to $40 \mathrm{mmHg}$ despite maximal medical therapy, and there was no elevation over the Ahmed plate (Figure 1). The patient's intense 

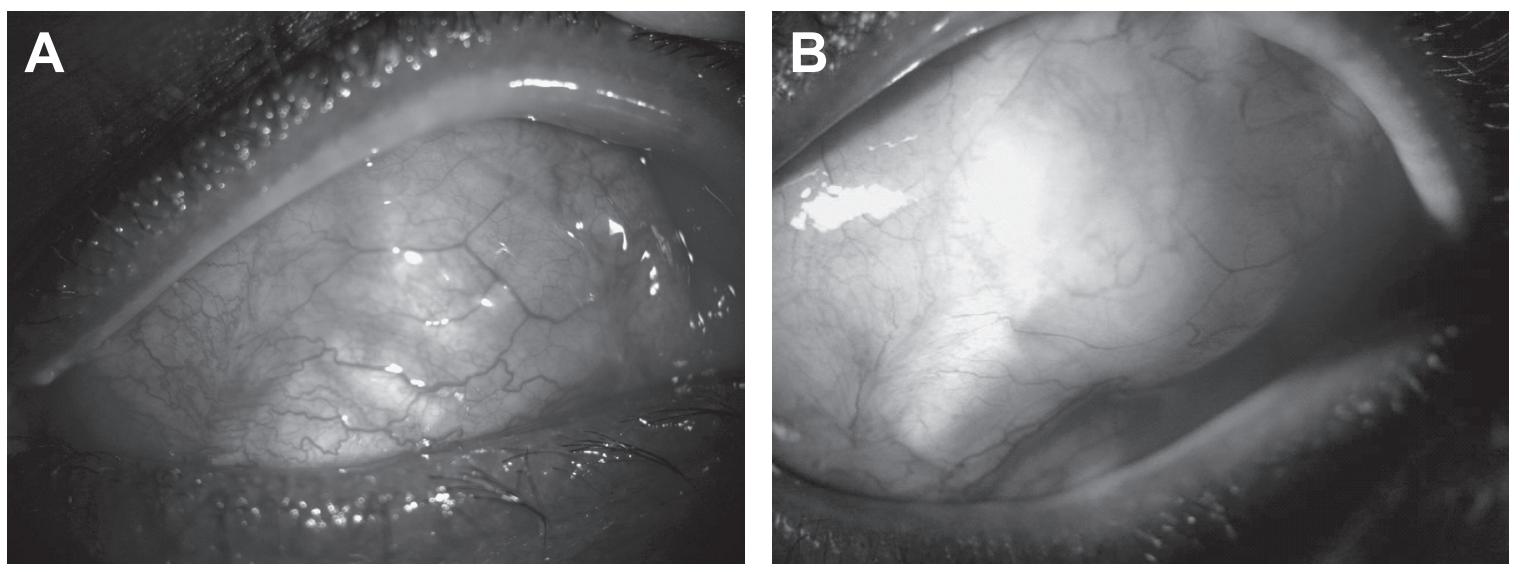

Figure I Slit lamp photographs of superotemporal bulbar conjunctiva of the left eye. (A) Before the intravitreal tPA injection, conjunctiva was flat over the Ahmed plate. Note the pericardium (Tutoplast, Costa Mesa, CA) and plate markings. (B) Two months following intravitreal tPA injection, there is a pseudocapsule over the functioning drainage device. Intraocular pressure is controlled with topical medications.

fibrinoid reaction in the anterior chamber, even on $100 \mathrm{mg}$ of oral prednisone daily, precluded an adequate view to the tube and suggested that fibrin was occluding the tube lumen.

Recombinant tissue plasminogen activator (tPA, Genentech Inc, South San Francisco, CA) was injected with a 30 -gauge needle $3.5 \mathrm{~mm}$ posterior to the limbus. A total of $0.1 \mathrm{cc}(12.5 \mu \mathrm{g})$ was injected. The next day, the IOP was $9 \mathrm{mmHg}$, and the bleb over the Ahmed plate was elevated. Five months later, visual acuity improved to $20 / 50$, and IOP was $17 \mathrm{mmHg}$ on brimonidine tartrate $0.15 \%$ twice daily and betaxolol $0.25 \%$ twice daily. The corneal graft was clear, a pseudocapsule had formed around the Ahmed plate and the tip of the Ahmed tube was well-visualized (Figure 2).

\section{Discussion}

Tissue plasminogen activator (tPA), a stimulator of fibrinolysis, has been used both subconjunctivally and intracamerally following glaucoma surgery since $1992 .{ }^{3}$ There are no prior reports of intravitreal tPA injection following glaucoma surgery.

Zalta and colleagues reported the largest study using intracameral tPA for occluded or near-occluded limbal glaucoma drainage devices with $89 \%$ of subjects $(n=36)$ achieving success, defined as avoiding the need for additional glaucoma surgery. ${ }^{4}$ In their study, patients received a mean of 1.6 injections with a mean tPA dose of $15.9 \pm 9.9 \mu \mathrm{g}$ per injection. Six patients had significant complications (severe hyphema, hypotony, anterior chamber flattening), which all occurred after injections that were administered within 5 days after glaucoma surgery.

An important consideration with a pars plana glaucoma tube after corneal surgery, as in our case, is that the tube tip may not be visible in the immediate postoperative period due to corneal edema and/or anterior chamber fibrin. Zalta and
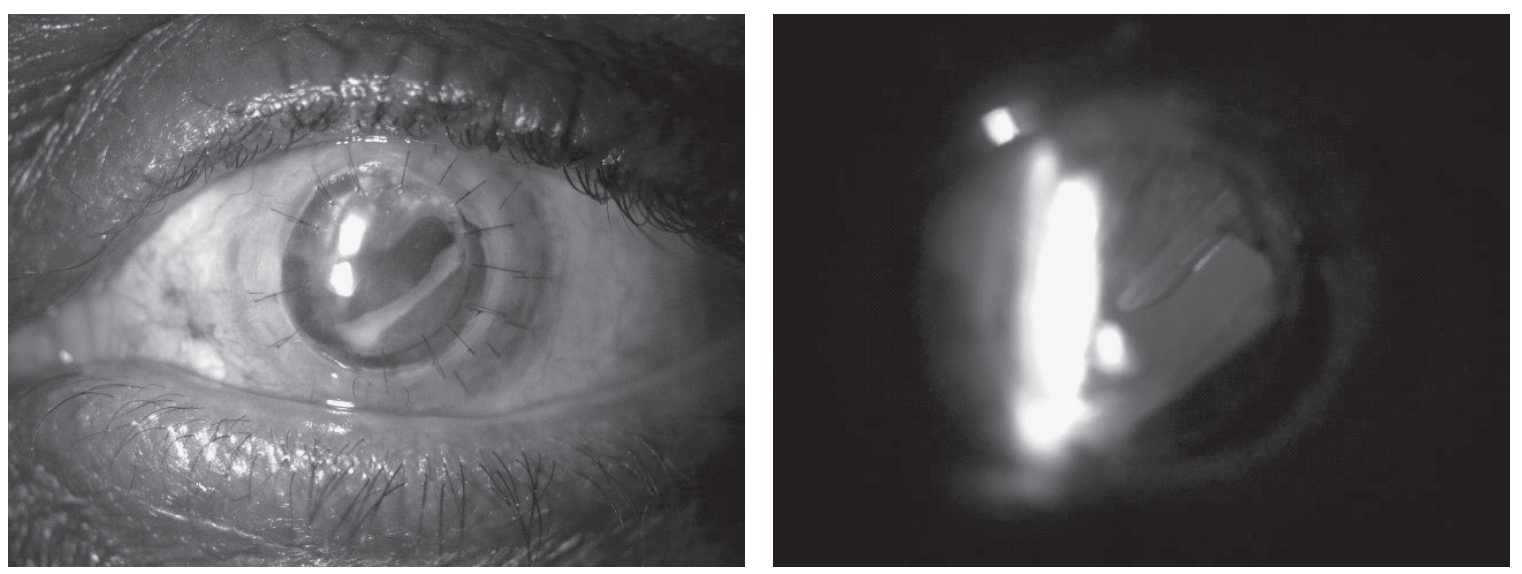

Figure 2 Slit lamp photographs of the left eye taken two months postoperatively. (A) Clear corneal graft with optic capture of a MA60AC (Alcon, Fort Worth,TX) intraocular lens. (B) The tip of the Ahmed tube in the anterior vitreous cavity is not occluded, as visualized by retroillumination. 
colleagues only used tPA on patients who had a visibly occluded or visibly near-occluded tube, defined as fibrin or blood within $2 \mathrm{~mm}$ of the tube tip.

Tissue plasminogen activator already has other intraoperative and postoperative uses for the retinal surgeon, such as in displacing submacular hemorrhages, reducing postvitrectomy fibrin formation, and maintaining patent inferior iridectomies in eyes with silicone oil. ${ }^{5}$ However, in both animal studies and one human case report, photoreceptor toxicity was noted following $50 \mu \mathrm{g}$ of intravitreal tPA. ${ }^{6,7}$

Our case demonstrates successful application of intravitreal tPA in a nonfunctioning pars plana Ahmed tube. Our patient achieved IOP control without further intraocular surgery. This innovation is particularly relevant because of the increasing frequency of implanting Ahmed tubes through the pars plana.

We conclude that intravitreal tPA is a useful option for patients with nonfunctioning pars plana glaucoma tubes due to fibrin. The procedure can avoid a reoperation on an already inflamed and vulnerable postsurgical eye. Future investigation should be directed towards the optimal effective and safe dose of intravitreal tPA, taking into consideration that the medicine will quickly leave the eye once patency of the glaucoma tube is reestablished.

\section{Acknowledgments}

Martha Moszczynski, BA and Rachel Revilla-Groh, BS; digital imaging specialists of the Department of Ophthalmology, Columbia University. The authors report no conflicts of interest in this work.

\section{References}

1. Al-Torbak A. Graft survival and glaucoma outcome after simultaneous penetrating keratoplasty and Ahmed Glaucoma Valve implant. Cornea. 2003;22:194-7.

2. Ritterband DC, Shapiro D, Trubnik V, et al. Penetrating keratoplasty with pars plana glaucoma drainage devices. Cornea. 2007;26:1060-6.

3. Szymanski A. Promotion of glaucoma filter bleb with tissue plasminogen activator after sclerectomy under a clot. Int Ophthalmol. 1992;16:387-90.

4. Zalta AH, Sweeney CP, Zalta AK, et al. Intracameral tissue plasminogen activator use in a large series of eyes with valved glaucoma drainage implants. Arch Ophthalmol. 2002;120:1487-93.

5. Kamei M, Estafanous M, Lewis H. Tissue plasminogen activator in the treatment of vitreoretinal diseases. Semin Ophthalmol. 2000;15:44-50.

6. Chen SN, Yang TC, Ho CL, et al. Retinal toxicity of intravitreal tissue plasminogen activator: case report and literature review. Ophthalmology. 2003;110:704-8.

7. Hrach CJ, Johnson MW, Hassan AS, et al. Retinal toxicity of commercial intravitreal tissue plasminogen activator solution in cat eyes. Arch Ophthalmol. 2000;118:659-63. 
